\title{
Long-Term Working Memory in the Rat: Effects of Hippocampally Applied Anisomycin
}

\author{
Sheri J. Y. Mizumori and Mark R. Rosenzweig \\ University of California, Berkeley \\ Edward L. Bennett \\ Melvin Calvin Laboratory \\ University of California, Berkeley
}

\begin{abstract}
The extent to which protein synthesis is involved in working memory was investigated with the protein synthesis inhibitor anisomycin (ANI). Rats were trained to perform accurately on a 12 -arm radial maze when delays of 240 min were interposed between choice 6 and choice 7 . Bilateral hippocampal cannulas were then implanted. Accuracy on choices 7-12 was studied when ANI or saline was injected either $30 \mathrm{~min}$ before choice 1 or 5-10 min after choice 6 (Experiment 1). Pretrial injection of ANI significantly impaired performance following the 240 -min delay, whereas ANI injected during the delay had no such effect. In Experiments 2 and 3, the ANI-induced amnesia was replicated, and the temporal course of development of the amnesia was determined. Pretrial administration of ANI did not significantly affect retention after a 2 -min delay but did produce amnesia after delays of $15 \mathrm{~min}$ or longer. These data suggest that protein synthesis is important for the formation of temporary memories, provided the retention interval is long enough. It is suggested that working memory includes both short-term and long-term components. Protein synthesis appears to be important for formation of the long-term component, but not the short-term component, of working memory.
\end{abstract}

The current literature shows that there is no generally accepted theory describing the neurochemical bases of learning and memory. An example is the lack of agreement on exactly what different forms of memory exist. Several dichotomies of memory have been proposed, for example, working and reference memory (Olton, 1983) and episodic and semantic memory (Tulving, 1972), most of which have been formulated from behavioral observations of humans or animals. Whether these behavioral distinctions actually represent quali-

This research was supported by National Institute of Mental Health Grant 1 R01 MH36042 and by the Department of Energy, Office of Energy Research, Office of Health and Environmental Research, under Contract DE-AC03-76SF0098.

We thank Michael G. Kermisch, Tracy Boisoneau, Carol A. Whitlatch, John Chia, Veena Channon, and Matthew R. Pease for assistance with behavioral testing. We also greatly appreciate the technical assistance of James G. Canfield and Arthur Deuberry.

Requests for reprints should be sent to Sheri J. Y. Mizumori, Department of Psychology, University of California, Berkeley, California 94720. tative differences in neurochemical function remains to be determined. In this study, we provide evidence that the neurochemistry underlying some of the proposed behavioral distinctions is indeed qualitatively different.

In animal investigations of memory, the distinction between short-term memory (STM) and long term memory (LTM) has often been emphasized. In studies on the role of protein synthesis in memory formation, it has been proposed that protein synthesis is not important for normal STM whereas the formation of new LTMs is protein synthesis dependent (e.g., Cohen \& Barondes, 1968). This conclusion is supported by experiments involving not only passive and active avoidance learning with rodents (e.g., Cohen \& Barondes, 1968; Davis, Rosenzweig, Jones, \& Bennett, 1981) but also rodent appetitive learning (Daniels, 1972) and avian avoidance and appetitive learning (Gibbs \& Ng, 1976, 1978). In these experiments, the temporal course of development of amnesia following the administration of specific classes of 
drugs is the main criteria for defining STM or LTM. On the other hand, Gibbs and Ng $(1979,1984)$ proposed that behaviorally significant phases in memory formation can be inferred from test performance of chicks. It was reasoned that if there are multiple, sequentially dependent memory phases, one should observe dips in performance level in untreated chicks at times when the transitions between phases occur. Indeed, such performance reductions were observed at times predicted by earlier neurobehavioral studies.

One difficulty with the interpretation of the experiments described above in which the chemical nature of STM or LTM was investigated is that although all of the experiments demonstrated that different neurochemical processes are involved in memory formation, none demonstrated directly that these phases of memory formation actually represent different functional (i.e., cognitive) stores, or processes, of memory. In order to establish the functional significance of the proposed neurochemical phases to memorial processes per se, more direct tests should be conducted, with tasks that allow a clearer behavioral definition of the kinds of memory under investigation. Delayed response tasks are often employed for this purpose. In particular, the radial maze (Olton \& Samuelon, 1976) has proved useful in determining operational characteristics of different kinds of memories. During radial maze training, rats are trained to retrieve food from the end of a number of alleys (or arms) that radiate from a center platform. The optimal strategy to use is to enter each alley only once during a given training session.

Olton and collaborators (Olton, 1978, 1983; Olton, Handelmann, \& Walker, 1981) described two kinds of memory that can be measured in the radial maze: working memory and reference memory. Reference memory contains more general information than working memory; it includes associations made regarding the rules and procedures necessary for appropriate interaction with one's environment (Honig, 1978; Olton, 1983). Therefore, reference memory items can be used on many occasions and can be stored for relatively long periods of time.
Items in working memory, on the other hand, are more specific in nature in that they have a "specific [i.e., unique] personal and temporal context to them" (Olton, 1983 , p. 338). Such items are applied to only one occasion and are typically not stored for long periods of time. According to the view that memory is a set of instructions (Honig, 1978), the instructions held in working memory are terminated when items are either called out of memory or are no longer needed.

A rat can demonstrate reference memory in a radial maze if it remembers from day to day the rules necessary to solve the maze. When the start door is raised, the rat readily runs down to the end of an arm in search of food. When the food is consumed, the rat quickly returns to a center platform where selection of an additional arm is made, and so on until all available arms have been chosen. The rules and set of environmental cues necessary for solving the maze are retained in reference memory.

Working memory can be shown and tested in the radial maze in the following way. The rat is allowed to retrieve food from only half of the total number of arms available. Following its selection of these choices, the rat is removed from the maze for a predetermined length of time. Retention of arms chosen before removal from the maze is thought to involve working memory. When a trained rat is placed back in the maze, it will enter only those arms not chosen before the delay period. Because rats vary the order of selection of arms from trial to trial, the items to be remembered over such a delay interval vary from trial to trial. Consequently, the set of cues governing the appropriate response also varies from trial to trial. Because of this, one can repeatedly examine characteristics of working memory in the same rat.

By directly comparing the neurochemical bases of STM and LTM with those of working and reference memories, one can obtain information regarding the functional significance of the proposed STM-LTM distinction in animals as well as information regarding the chemical basis of working and reference memories. These two dichotomies were chosen for comparison because of the 
proposed similarities between them; for example, both dichotomies have a temporal component: STM and working memory are of shorter duration than LTM and reference memory. In addition, working memory and STM are thought to be more vulnerable to disruption than reference memory and LTM (Honig, 1978; Wagner, 1978). Therefore, we began assessing the functional significance of the temporal stages of memory function, as defined by investigators such as Davis et al. (1981), by determining the effects of the protein synthesis inhibitor anisomycin (ANI) on working and reference memories. If the neurochemistry of working memory is analogous to STM, ANI should have no effect on retention of items stored in working memory. On the other hand, if working memory is more like LTM, ANI should impair retention of working memory items. Finally, it is possible that working memory includes both short- and long-term components. In this case, the amnesic effect of ANI should be dependent on the time of test relative to time of training.

\section{General Method}

Methods common to the three experiments in this report are described in this section. Procedures that are different are described individually for each experiment.

\section{Subjects}

Seven days after arrival in the laboratory, adult male rats were placed on a restricted food diet, with free access to water, so that their body weights were maintained at $80 \%-85 \%$ of their ad-lib body weights. Behavioral testing took place during the dark phase of the light/dark cycle, between 0800 and 1400 . Lights were on between 1900 and 0700 .

\section{Apparatus}

The rats were tested on an elevated radial maze ( 61 $\mathrm{cm}$ above the floor) similar to the one described by Olton and Samuelson (1976) except that 12 arms (80 $\times 10.2 \mathrm{~cm}$ ) extended from the center platform $(40.6$ $\mathrm{cm}$ in diameter). The door separating the arms from the center platform consisted of a single circular piece of sheet metal. The door could be raised or lowered by the experimenter by a system of fishlines from a corner of the room. Several items, such as chairs, doors, wall panels, water pipes, air vents, and posters, were available to serve as room cues.

\section{Drug Preparation}

Anisomycin (2-p-methoxyphenyl-3-acetoxy-4-hydroxypyrollidine) was obtained from Pfizer Pharmaceutical Company (now obtainable from Warner-Lambert, General Diagnostics Division). It was dissolved in saline by adding equal molar amounts of $3 \mathrm{~N} \mathrm{HCl}$. The $\mathrm{pH}$ was adjusted to $6-7$ by adding $\mathrm{NaOH}$. The same concentration of ANI $(80 \mu \mathrm{g} / \mu \mathrm{l})$ was used for all experiments in this report. The selection of this concentration was based on experiments reported by Grecksch, Ott, and Matthies (1980) and Grecksch and Matthies (1980), demonstrating this to be effective for altering memory function. In addition, it was shown that $80 \mu \mathrm{g}$ per hippocampus resulted in $91 \%-94 \%$ protein synthesis inhibition of hippocampal proteins $50 \mathrm{~min}$ after injection (Grecksch et al., 1980). That significant protein synthesis inhibition occurg earlier than 50 min is suggested by results of Kesner, Partlow, Bush, and Berman (1981). In their study, maximum inhibition by a similar protein synthesis inhibitor, cycloheximide, occurred by $30 \mathrm{~min}$ after injection into the amygdala. In preliminary studies in our laboratory, protein synthesis inhibition was measured $30 \mathrm{~min}$ after ANI was injected into the CA3 subfield of the hippocampus. Results indicate that protein synthesis inhibition in hippocampus was approximately $70 \%$. The inhibition caused by ANI showed a gradient, declining to about $20 \%$ in areas most distant from the site of injection. Because the hippocampus sample included more than the target CA3 subfield, inhibition in the target area may well have been higher than $70 \%$.

\section{Surgical Procedures}

Rats were injected ip with $75 \mathrm{mg} / \mathrm{kg}$ sodium pentobarbital. In order to guard against possible infection from the surgery, Bicillin $(0.05 \mathrm{ml})$ was given im to the left hind leg. Two stainless steel guide cannulas (23 ga.) were implanted bilaterally into the posterior portion of the CA3 subfield of the hippocampus according to the following stereotaxic coordinates: A-P $-4.2, \mathrm{~L} \pm 4.8, \mathrm{D} \cdot \mathrm{V}-6.0$. That the CA3 subfield is involved in spatial memory, as demonstrated in the radial maze, has been reported previously (e.g., Handelmann \& Olton, 1981). The guide cannulas were embedded in dental acrylic which was anchored to the skull with two No. 0-80 stainless steel screws.

For drug administration, rats were lightly anesthetized with Metofane, then placed in a stereotaxic instrument. Injection needles ( $31 \mathrm{ga}$.) were lowered into the guide cannulas such that the tip of each needle was $6.3 \mathrm{~mm}$ below the outer surface of the skull. A volume of $0.5 \mu \mathrm{l}$ per hippocampus of ANI or saline (SAL) was delivered over a period of $88 \mathrm{~s}$ by an automatic syringe pump. Thirty seconds later, the injection needles were removed, and the rat was placed back in its home cage.

After behavioral testing was completed, the brains were removed for histological verification of cannula placement. Frozen sections ( $40 \mu \mathrm{m}$ thick) were stained with cresyl violet, then examined under a light microscope. 


\section{Experiment 1}

\section{Method}

Subjects. Male albino rats were obtained from the Psychology Department of the University of California, Berkeley at approximately 90 days of age.

Training procedure. One day after food restriction began, daily 10 -min periods of adaptation to the maze were given by placing each rat on the maze with 45 mg Noyes pellets scattered about. Following 2 days of adaptation, daily $10-$ min shaping trials began. A rat was placed on the center platform. Ten seconds later, the door was raised, which allowed the rat to move freely in the maze. Five 45-mg Noyes pellets had been placed in cups at the end of each arm. In addition, one Noyes pellet had been placed at the entrance of each arm and another halfway down the arm. The specific arms entered, the distance traveled down each arm, whether food pellets were consumed, and the time required to reach the end of each arm were recorded. This shaping procedure continued until the rat went all the way down each arm entered and entered at least 12 arms in $10 \mathrm{~min}$.

The training procedure was similar to the shaping procedure except that the only food available on the maze was two Noyes pellets placed in each cup at the end of each arm. Training continued until a rat reached criterion performance levels, that is, made at least 11 correct (different) choices in the first 12 choices of the training session and chose all 12 arms within the first 14 choices for 7 consecutive days. In addition, the first 12 choices had to be made within 5 min. The day after a rat met this criterion, delay training began: After the rat made 6 choices, it was confined to the center for $1 \mathrm{~min}$, after which time the door was raised and the rat was allowed to complete the session. When the rat reached delay criterion performance level, that is, made at least 5 correct choices in the first 6 choices following the delay and chose the remaining correct arms within the first 8 choices after the delay for 1 day, it was advanced to 2 -min-delay training. During this delay, the rat was removed from the maze, placed in its home cage for the duration of the delay, then placed back in the maze to complete the session. When the same criterion performance was met with 2-min delay as was met with the 1-min delay, each rat was trained to maintain information in working memory for even longer periods of time. Instead of using the more common technique of training rats with progressively longer delay intervals, we chose to train the rats according to a blocked delay training procedure (outlined below). We used this technique because we were interested in determining how long the memory of choices 1-6 would normally last when a rat had not been explicitly trained to maintain items in working memory for a long period of time. With continued training, we were also able to monitor the development of a working memory for longer durations.

During the first 9-day block of delay training, a rat was run once with each of eight delay intervals. The order of delay intervals used was different for each rat and was determined in a quasi-random manner. The delay intervals used were $0,15,30,60,120,180$, and $240 \mathrm{~min}$ and $24 \mathrm{hr}$. For subsequent blocks of 9 days, each rat was assigned a different order of delay intervals. Each rat underwent at least four 9-day blocks of delay training; such delay training ended when a rat performed at criterion levels at all delays (except 24 hr) within an entire block. Rats were not trained to criterion with $24-\mathrm{hr}$ delays because we wanted the rats to retain information for only a few hours. The 24-hr test was used here to demonstrate that there was no proactive effect of one day's choices on successive training days. Finally, in order to make it clear that the rat had mastered 240 -min delays, each rat was run with only 240 -min delays until criterion performance was met for 3 successive days.

After each rat reached criterion performance with 240-min delays, it was given free access to food for 7 days. On the eighth day, food was removed. The next day, an intrahippocampal cannula assembly was implanted, as described above. Following surgery, the rat was given free access to food for 7 days after which food was removed and postsurgical training began. Postsurgery training was identical to the blocked delay training procedures outlined above except that such training ended as soon as a rat performed at criterion levels for all delays less than $24 \mathrm{hr}$ within an entire block. Finally, rats were run with only 240 -min delays until criterion performance was shown for 3 successive days.

During the drug injection phase of the experiment, the rats were injected with either SAL or ANI within 5 to $10 \mathrm{~min}$ after choice 6 or $30 \mathrm{~min}$ before choice 1 . The effects of 5-10-min posttrial injections were tested first. Seven days after the first set of injections, rats that previously received SAL were injected with ANI after choice 6; rats that previously received ANI were injected with SAL after choice 6 . All retention tests occurred following a 240 -min delay. Shorter delays were not used in Experiment 1 because we first wanted to establish whether ANI had an effect on memory over a delay; using the longest delay would maximize the chances of observing an effect. Two weeks after the rats received the first two injections, SAL or ANI was again injected in a counterbalanced order, but this time injections occurred $30 \mathrm{~min}$ before choice 1. Retention testing occurred following a 240min delay. The last two injections were given about 1 week apart.

\section{Results and Discussion}

Each rat underwent an average of 10.4 days of shaping before training. This was followed by a mean $\pm S E$ of $42.1 \pm 2.4$ days of training. Delay training with 1 - and 2min delays required a mean of 2.9 days before criterion performance was met. The rats reached criterion performance with delay training after $41 \pm 4.1$ days. Figure 1 demonstrates that as the number of delaytraining blocks increased, there was a progressive increase in choice accuracy follow- 


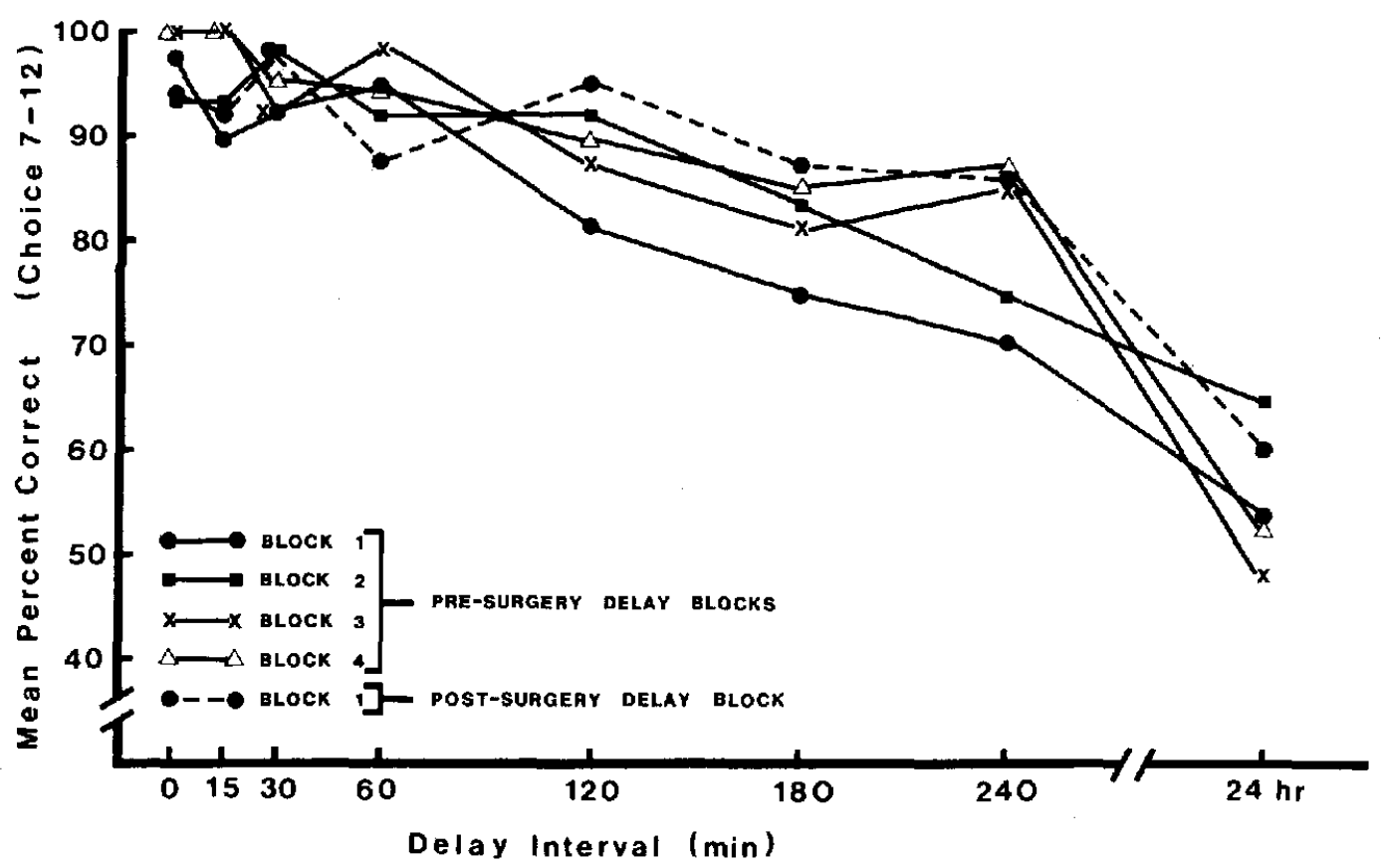

Figure 1. Choice accuracy following variable delay intervals $(n=8)$. (Presurgical delay Block 1 represents the earliest stage of training; Block 4 is the last block before surgery. Postsurgical delay Block 1 is the first series of delay training following surgery.)

ing long delays (120-240 min). Two-variable repeated measures analysis of variance (Keppel, 1982) revealed a significant improvement in overall performance from presurgical delay Block 1 to Block $4, F(1$, 7) $=22.91, p<.01$. In addition, accuracy decreased significantly as delay length increased, $F(7,49)=20.91, p<.001$. There was no interaction of delay interval length and block number, $F(7,49)=1.26$, ns. The significant main effect of block number appeared to be due to the improvement in choice accuracy when 240-min delays were used, $F(1,7)=9.33, p<.05$.

These results demonstrate that extended delay training was not necessary for rats to retain items in working memory for up to $1 \mathrm{hr}$. However, further training was necessary for items to be remembered for longer periods of time. Figure 1 shows that the memory lengthened progressively as training continued.

Choice accuracy following a 24-hr delay never improved during the four blocks of training. This suggests that information regarding the first six choices was held in memory only temporarily, that is, for a few hours. Choice accuracy following a $24-\mathrm{hr}$ delay was significantly lower than that after a 240-min delay, even during the first block of delay training, $t(7)=2.49, p<.05$. It appears that there was some form of memory for choices 1-6 after a delay of 240 min in the first block of training, but, relative to the maximum level of performance that rats demonstrated at short delays, retention at $240 \mathrm{~min}$ was relatively poor. Performance following the 24-hr delay was only slightly above that expected by chance alone, which is $40.2 \%$ correct.

Following training with delay blocks, rats required 3.5 days (including the 3 criterion days) to reach criterion at 240 -min delays. Postsurgical training typically required one or two blocks of delay training before criterion performance was met. Group mean performance for the first block of training after surgery did not significantly differ from the last block of delay training before surgery (see Figure 1). Following criterion performance with postsurgical delay block training, the rats performed at maximal 


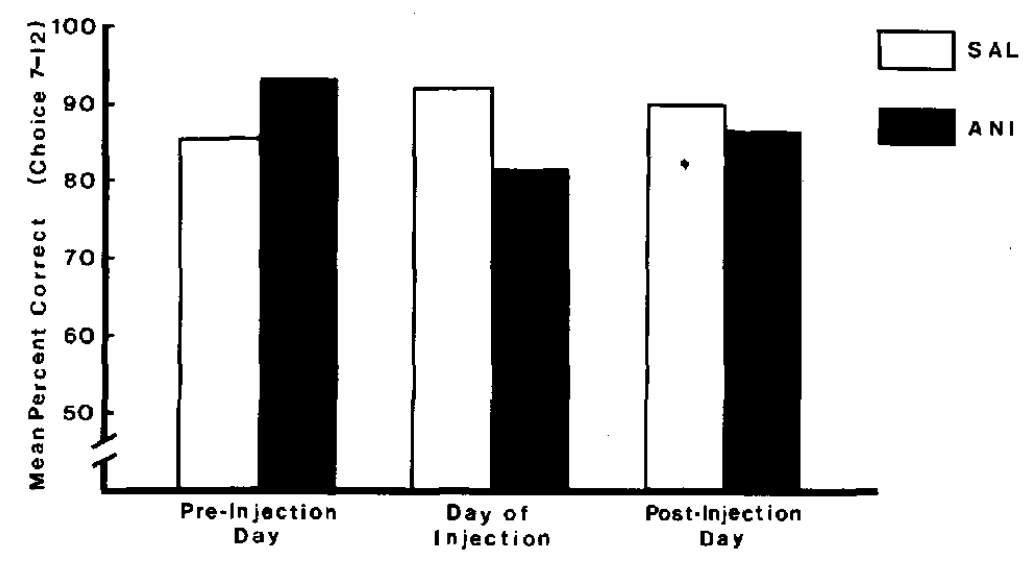

Figure 2. Choice accuracy following 240-min delays. (Anisomycin [ANI] or saline [SAL] was injected within 5 to $10 \mathrm{~min}$ after choice 6 . Group $n s=8$. There are no significant differences.)

levels when tested following only 240 -min delays; a mean of only 3.0 days was necessary to reach criterion.

Anisomycin injected at the beginning of a 240 -min delay, 5-10 min after choice 6 , had no significant effect on retention following the delay. Figure 2 illustrates that this was true when such scores were compared with retention scores following SAL injection, $t(7)=1.21, n s$, or when compared with the same rat's previous day's noninjected retention score, $t(7)=1.57$, ns. Saline injections had no significant effect on retention over a $240 \mathrm{~min}$-delay relative to the previous day's noninjected score, $t(7)$
$=1.07$, ns. Two-variable analysis of variance revealed no significant interaction of drug effect and data from the day before injection and day of injection, $F(1,28)=$ 3.61 , ns. Figure 2 also shows that neither SAL nor ANI had proactive effects on the next day's retention over a 240 -min delay, $t(7)=0.68$, ns and $t(7)=0.55, n s$, respectively.

In contrast to the lack of effect of ANI injected during the delay interval, ANI injected $30 \mathrm{~min}$ before choice 1 resulted in significant impairment of memory following a 240-min delay (see Figure 3). The impairment was significant whether com-

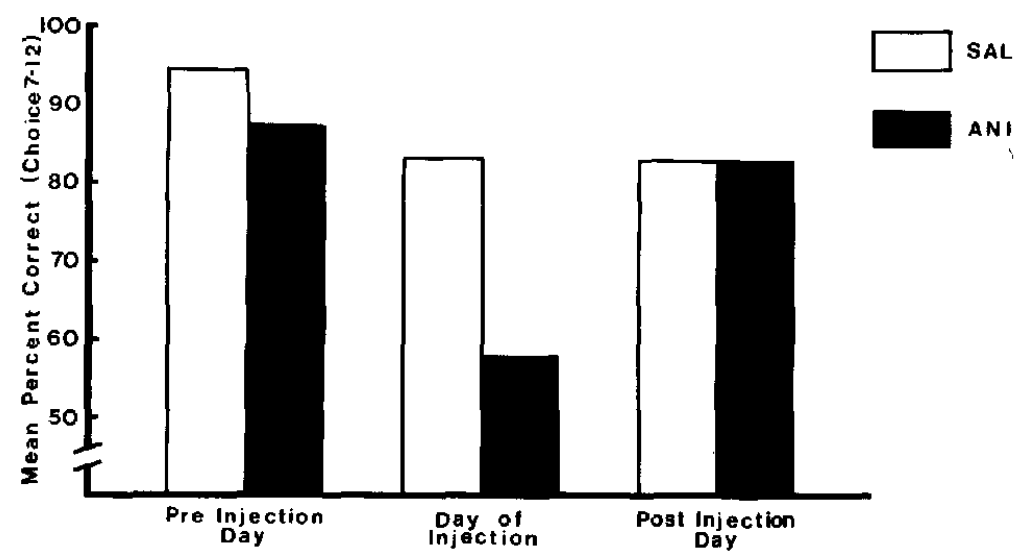

Figure 3. Choice accuracy following 240-min delays. (Anisomycin [ANI; $n=4$ ] or saline [SAL; $n=$ 3] was injected $30 \mathrm{~min}$ before choice 1 . Significant impairment of memory was observed when performance of ANI-treated rats was compared with that of SAL-injected rats on control days or on the day before ANI was injected.) 
parisons were made with scores of SALinjected control days or with the same animal's previous day's noninjected score, $t(5)$ $=2.93, p<.05$ and $t(3)=4.70, p<.05$, respectively. Saline injections $30 . \mathrm{min}$ before choice 1 did not impair retention after a delay, $t(2)=1.00, n s$, nor did SAL or ANI injections affect performance on days following the injection, $t(2)=1.07$, $n s$ and $t(3)=0.52$, ns, respectively. Because of technical difficulties with the implanted cannulas (mainly plugged cannulas), which occurred after the first phase of this experiment, 4 animals were not included in the second phase of the experiment. Accurate cannula placement was verified histologically for all rats that contributed data to Figures 2 and 3 . It should also be noted that the ANI injections did not produce any obvious behavioral side effects such as sickness or motor deficiencies. In addition, the amnesic effect was not dependent on the order of injection of ANI and SAL.

That pretraining injections of ANI significantly impaired memory suggests that protein synthesis is important for good retention over long delays. As shown in previous experiments with protein synthesis inhibitors (e.g., Bennett, Rosenzweig, \& Flood, 1977), the time of injection relative to the time of initial acquisition of information is critical for the protein synthesis inhibitor to be an effective amnesic agent; that is, ANI given before training produced amnesia more reliably than posttraining injections. In addition, previous experiments showed that protein synthesis is important for permanent LTMs to be formed. Here, we show that relatively short lasting, temporary memories may, under certain conditions, also require the synthesis of new proteins. However, because all injections of ANI before choice 1 were given to rats that had previously received ANI (during the delay), the possibility that the second injection of ANI was effective because of heightened sensitivity to, or some other subtle proactive effect of, the first injection of ANI could not be ruled out. A second experiment was therefore carried out to test the replicability of the amnesia observed in Experiment 1 and also to determine the temporal course of development of the amnesia.

\section{Experiment 2}

\section{Method}

Subjects. Seventy-day-old male Long-Evans rats were obtained from Simonsen's Breeding Laboratory (Gilroy, California). The strain and supplier of subjects were changed in this experiment because of a limited supply of rats in our department.

Training procedure. Maze adaptation and shaping procedures were carried out as described in Experiment 1 . Predelay training in the maze also followed the same procedure as described above. Delay training, however, was carried out in a slightly different manner than that used in Experiment 1 because it was thought that the delay training phase of the experiment could be shortened. In addition, as the procedure used here is the one more typically used in other radial maze experiments with long delay intervals, the results may be more comparable to results of other experiments. The revised delay training procedure involved training rats to perform correctly with progressively longer delay intervals. This is in contrast to training rats according to the blocked training described in Experiment 1. Specifically, in Experiment 2, a rat was first trained to criterion performance (as defined in Experiment 1) at 1-min delays, then at delays of $2,15,30$, $60,120,180$, and $240 \mathrm{~min}$, in that order. During the 1min delay, the rat was confined in the center of the maze. For longer delays, the rat was returned to its home cage for the duration of the delay. An additional 3 successive days of criterion performance was required with 240 -min delays before presurgical ad-lib access to food was allowed. Following 7 days of free access to food, food was removed. Then, the cannula assembly was implanted. Food was replaced for 7 days after cannula implantation. On the eighth day, food was removed and postsurgical training began.

In postsurgical training, the rat was first run in the maze with no delay. When criterion performance was met for 1 day, delay training began. A rat advanced to progressively longer delays when criterion performance was met for 1 day at each delay interval. When a rat was trained with a $240-$ min delay, 3 successive days of criterion performance were required before drug injections began.

During the drug injection phase of the experiment, rats were injected with ANI or SAL 30 min before choice 1 , then tested for retention of choices 1-6 following $0-, 30-, 60-, 120-$, or 240 -min delays. When a rat was tested following a 0 -min delay, no confinement was imposed between choice 6 and choice 7 ; that is, the rat was allowed to complete the maze uninterrupted by the experimenter. It should be pointed out that although the rat was allowed to run continuously through the maze, the rat necessarily had to retain information regarding the selection of earlier choices until all correct choices had been made. Regardless of drug treatment, it typically took a rat 4-5 min to choose all 12 correct arms when no delay was imposed. Each rat was assigned to different delay intervals for both ANI and SAL injections in a quasi-random order. Following drug injection, a given rat was required to perform at criterion levels for 3 successive days before it was injected again. 


\section{Results and Discussion}

A mean of 8.2 days was required to shape the rats to the maze. Training to criterion with no delays required a mean $\pm S E$ of 15.4 \pm 1.9 days, which included the 7 successive criterion days. The rats readily learned to retain choices 1-6 in memory for progressively longer periods of time; almost all of the rats required only 3 days (near-perfect performance) to reach criterion performance at each of the delay intervals. Therefore, a rat typically reached criterion in 24 days. This compares with 41 days required by rats trained according to the block procedure of Experiment 1. Results from postsurgical training clearly indicated that, as was demonstrated in Experiment 1, cannula implantation did not affect the rats' performance in the maze. In particular, criterion performance for almost all of the rats was achieved on the first day of training as well as on the first day of each delay interval used.

Table 1 shows that ANI impaired retention following all delay intervals tested. Analysis of variance for repeated measures showed a significant effect of drug treatment, $F(1,4)=23.44, p<.01$. No difference was observed for retention as a function of delay interval length, $F(4,16)=1.86$, ns. Similarly, there was no significant interaction of delay length and drug treatment, $F(4,16)=0.94, n s$. When choice accuracy was tested with no delay period between choice 6 and choice 7, ANI did not impair retention, $t(4)=1.00, n s$. In contrast, when ANI-injected rats were tested for retention following $30-, 60-, 120-$, or 240-min delays, significant impairment was observed relative to days when the same rats were injected with SAL. These findings not only

Table 1

Mean Percentage of Correct Choices in Choices $7-12$

\begin{tabular}{lccccc}
\hline & \multicolumn{5}{c}{$\begin{array}{c}\text { Delay } \\
\text { interval } \\
\text { (in min) }\end{array}$} \\
\cline { 2 - 6 } Treatment & 0 & 30 & 60 & 120 & 240 \\
\hline Saline & 100 & 93.3 & 96.7 & 96.7 & 96.7 \\
Anisomycin & 93.3 & $70.0^{*}$ & $70.0^{* *}$ & $70.0^{*}$ & $73.3^{*}$ \\
${ }^{*} p<.05 . * *$ \\
\end{tabular}

replicate the impairment observed in ANItreated rats tested in Experiment 1 but indicate that the amnesia developed soon after choice 6 .

The number of errors, that is, repeated arm entries, made before the rat obtained all remaining food pellets after a delay was also determined (see Figure 4). Analysis of variance for repeated measures revealed a significant effect of drug treatment, $F(1,4)$ $=11.51, p<.05$, no effect of delay interval length per se, $F(4,16)=1.03, n s$, and no Drug $\times$ Delay interaction, $F(4,16)=1.13$, $n s$. Application of the $t$ test, however, revealed that whereas ANI injections resulted in a statistically nonsignificant increase in the number of errors made when the rats were tested after 0 or $30 \mathrm{~min}, t(4)=1.00$, and $t(4)=1.46$, respectively, a statistically significant increase in number of errors made was found when they were tested after delays of 60,120 , or $240 \mathrm{~min}$ (all $p$ s $<$ .05). This finding suggests that although the rats may have had some retention of earlier choices when tested 30 min after training, the residual memory was inadequate for demonstration of the usual high performance early in the test session. In contrast, amnesia of ANI-treated rats tested after 60 -, 120 -, or 240 -min delays was more severe.

Although no errors were made on choices 1-6 for either SAL- or ANI-treated rats, Table 2 shows that errors made following a delay were almost always due to revisits to arms entered on choices 1-6. In contrast, repeat visits to the remaining correct choices after a delay were minimal. This indicates that ANI had little or no effect on reference memory aspects of the task; the rats clearly attempted to solve the maze according to the rules learned previously. In addition, the fact that performance on days following injection was not impaired suggests that ANI did not permanently affect reference memory function. Finally, certain observations of choice behavior after the delay also suggest that reference memory was intact. At the start of a test session, rats readily ran down arms, investigated the food cup, then quickly turned around to return to the center platform. During the selection of the next choice, the characteristic head pokes through the en- 


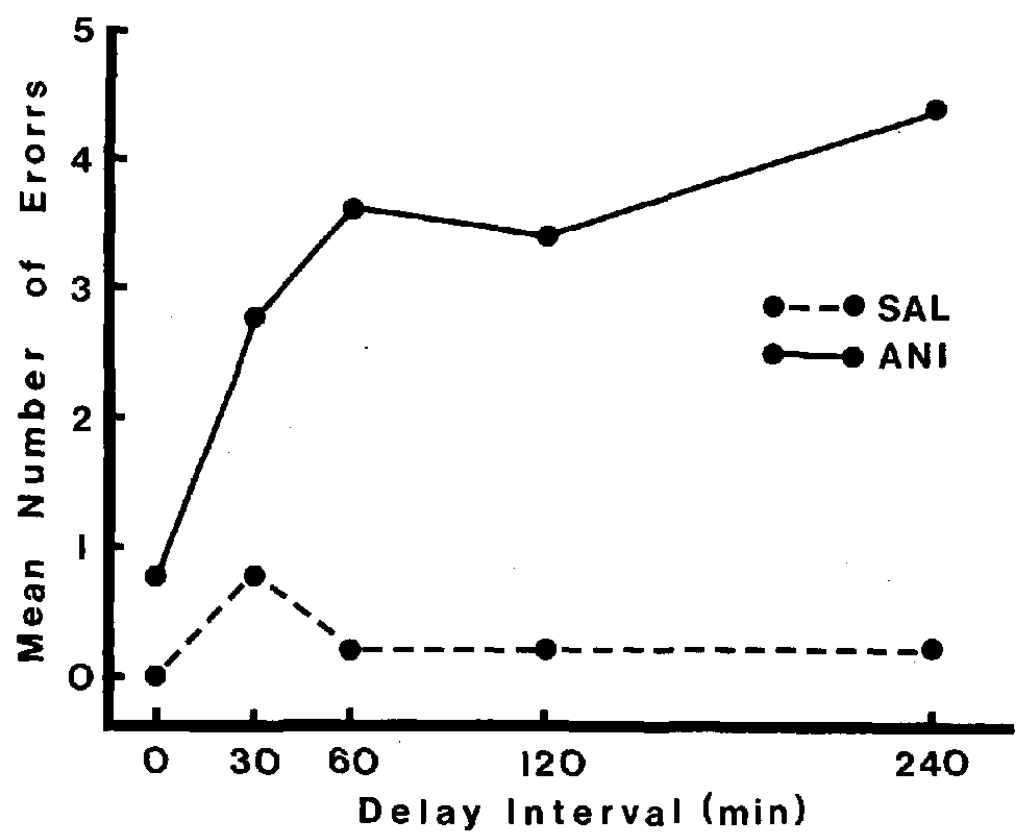

Figure 4. Total number of errors made before entering all 12 arms at least once. (Anisomycin [ANI] or saline [SAL] was injected $30 \mathrm{~min}$ before choice 1 . Variable delay interval lengths were imposed between choice 6 and choice 7.)

trance to the arms were observed. Also, as is typical of rat behavior after a delay, the rat did not always enter the arm on which the head poke occurred.

An alternative explanation for the impairment in choice accuracy described above is that ANI injections resulted in increased susceptibility to environmental stimulus interference caused by the extra handling involved when delays are imposed. In Experiment 3 we examined this hypothesis by testing rats after shorter de-

Table 2

Mean Number of Errors at Test

\begin{tabular}{lccccc}
\hline & \multicolumn{5}{c}{ Delay interval (in min) } \\
\cline { 2 - 6 } Treatment & 0 & 30 & 60 & 120 & 240 \\
\hline & \multicolumn{5}{c}{ Revisits to arms } \\
& \multicolumn{5}{c}{ entered on choices $1-6$} \\
Saline & 0.0 & 0.6 & 0.2 & 0.2 & 0.2 \\
Anisomycin & 0.6 & 2.6 & 3.6 & 3.4 & 3.8 \\
\hline & \multicolumn{5}{c}{ Revisits to remaining } \\
& \multicolumn{5}{c}{ correct arms } \\
Saline & 0.0 & 0.2 & 0.0 & 0.0 & 0.0 \\
Anisomycin & 0.2 & 0.0 & 0.0 & 0.0 & 0.6 \\
\hline
\end{tabular}

lays than those used in Experiment 2. If it could be demonstrated that ANI-treated rats have good retention with the shortest of delays $(2 \mathrm{~min}$ ), then it could be argued that an imposed delay per se does not cause an ANI-treated rat to show poor retention at test.

\section{Experiment 3}

\section{Method}

Subjects. Male Long-Evans rats were obtained from Charles River Breeding Laboratory (Wilmington, Massachusetts) at 70 days of age.

Training procedure. Rats were trained on the radial maze as described in Experiment 2. After they had achieved criterion performance with 240 -min delays, cannulas were implanted into the hippocampus. Following postsurgical training to criterion, rats were injected with either SAL or ANI $30 \mathrm{~min}$ before choice 1. Retention for choices 1-6 was tested after either a 2- or a 15-min delay. The order of drug treatment and delay interval used was counterbalanced.

\section{Results and Discussion}

The rats required $15 \pm 1.5$ days $(M \pm$ $S E)$ to train to criterion with no delay interval interposed between choice 6 and 
choice 7 , which included the 7 successive criterion days. Then, like the rats of Experiment 2 , these rats readily learned to retain information regarding choices 1-6 in memory for long periods of time. Typically, criterion performance was achieved after 3 days for each delay interval. During postsurgical training, criterion performance was almost always achieved on the first day of each delay interval used.

When ANI was injected $30 \mathrm{~min}$ before choice 1 , imposition of a 2-min delay between choice 6 and choice 7 had no significant effect on choice accuracy at test, $t(4)$ $=1.83$, ns. Mean percentages of correct choices for choices 7-12 were 83.3 and 100 for ANI and SAL groups, respectively. In contrast, ANI-treated rats showed marked impairment after a 15 -min delay relative to SAL-treated rats, $t(4)=4.02, p<.01$. Mean percentages of correct choices after a 15 min delay for ANI and SAL groups were 63.3 and 93.3, respectively. It appears that the process of removing a rat from the maze and placing it in its home cage does not, by itself, induce amnesia in ANI-treated rats. Whether amnesia is observed depends on the length of the retention interval.

\section{General Discussion}

Results from the experiments reported here clearly indicate a significant role for protein synthesis in the temporary storage of information, provided the retention interval is long enough. Anisomycin injected into the hippocampus before maze training impaired retention of working memory items when the rats were tested $15 \mathrm{~min}$ or longer after training. Figure 5 illustrates the time-dependent nature of the impairment, based on findings from Experiments 2 and 3. That amnesia develops rather quickly suggests that the initiation of memory-related protein synthesis must occur close to the time of initial acquisition if memories are to be held over a delay period.

Because ANI had no significant effect on choice accuracy when no delay or a 2-min delay was imposed between choice 6 and choice 7 , it appears that the information necessary for correctly solving the maze not only was perceived by the rat but also was

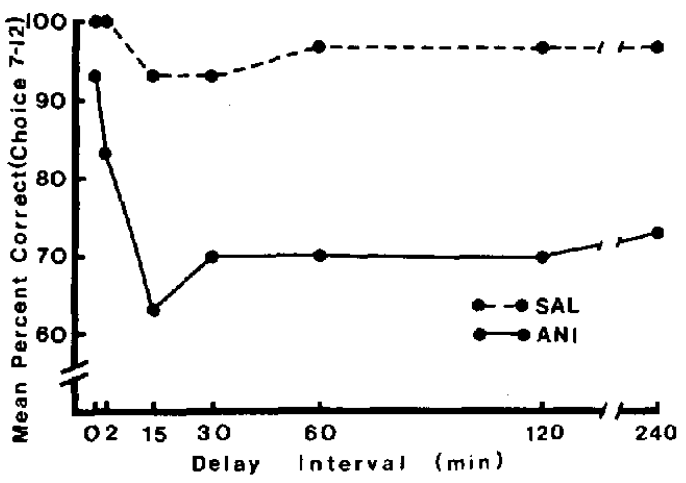

Figure 5. Combined summary of results from Experiments 2 and 3. (Anisomycin [ANI] injected $30 \mathrm{~min}$ before choice 1 significantly impaired retention following delays of 15 min or longer. Retention of ANItreated rats after a 0 - or 2-min delay was not significantly different from retention of saline [SAL] treated rats after similar delays.)

utilized in an efficient manner. The acquisition and short-term maintenance of such information cannot therefore be protein synthesis dependent. However, as imposition of even a 15-min delay resulted in impairment, it appears that the transfer of information from a short-term to a longterm working memory involves the synthesis of new proteins. Furthermore, as ANI injected during the delay had no effect on subsequent retention (see results of Experiment 1), it appears that the maintenance process per se within working memory is not protein synthesis dependent. Because ANI appeared to have no effect on reference memory aspects of the task, we conclude that protein synthesis is not necessary for maintenance, and subsequent utilization, of reference memory.

The proposed role for protein synthesis in working memory function outlined above does not necessarily indicate that protein synthesis is involved in STM. Rather, another explanation seems more plausible: Working memory has both short- and longterm temporal components. Thus, working memory and STM are not one and the same. We therefore assert that protein synthesis is important for formation of the long-term, but not the short-term, component of working memory. That working memory contains a long-term component has also been suggested by Bierley, Kesner, 
and Novak (1983). Using the radial maze, these investigators demonstrated that subseizure level stimulation of the dorsal hippocampus resulted in impairment of retention following 20 -min delays but not 1 - or 12 -min delays. They concluded that the hippocampus is important for long-term working memory but not for STM. Consistent with this interpretation of hippocampal function is the report by Rawlins and Tsaltas (1983). In that experiment, rats with hippocampal lesions performed well on a delayed alternation lever-press task when the delays were relatively short $(3.75$ $\mathrm{s})$. When longer delays $(12.75 \mathrm{~s})$ were imposed, however, impaired performance was observed. Rawlins and Tsaltas concluded that the hippocampus is involved in what they called "intermediate-term" working memory but not short-term working memory. The results of the present study are in accord with both the hypothesis suggested by Bierley et al. and that of Rawlins and Tsaltas. In addition, we suggest that STM processes might be involved in the selection of choices before a delay.

Other evidence exists that suggests working memory is not the same as STM. Spatial working memory, as demonstrated in a radial maze, for example, is resistant to disruption by stimuli that disrupt STM in other experimental paradigms (e.g., Maki, Brokofsky, \& Berg, 1979). Furthermore, Klapp, Marshburn, and Lester (1983) suggested that human working memory and STM are not the same; in brief, they provided evidence that working memory contains a greater information-processing capacity than STM.

The short-term aspects of working memory could include processes responsible for information acquisition/registration and subsequent utilization when it is not required that the information be retained for long intervals. In contrast, the long-term component is responsible for the maintenance, although only temporarily, of items in working memory over prolonged periods of time. What determines the specific temporal course of working memory may be the nature of the items to be remembered (Honig, 1978) as well as the particular training procedures used. As other re- searchers have noted, rats trained to perform in the radial maze, especially with very long delay intervals, may be considered to be somewhat overtrained. The overtraining may have created a situation in which different components of working memory were readily distinguishable, perhaps by increasing the speed with which information is transferred from short-term to long-term working memory. With extensive training, the rats in this study learned that information regarding choices $1-6$ should be retained in memory for several hours, although still only temporarily, in a form that was not vulnerable to disruption by exposure to background auditory or visual noise. More specifically, the rats may have learned that the lowering of the doors after choice 6 was a cue to store information in a long-term working memory. That memory processes in the rat can be brought under external stimulus control has indeed been demonstrated by Grant (1982). Similarly, Agranoff (1967) suggested that the effectiveness of protein synthesis inhibitors is dependent on environmental cues signaling LTM formation processes to start.

It has been shown that when rats are trained in the radial maze, they can retain information in working memory for up to 6 hr (Shavalia, Dodge, \& Beatty, 1981) in the radial maze. It has also been suggested that tests conducted $6 \mathrm{hr}$ after avoidance training reveal the functional integrity of LTM (e.g., Davis et al., 1981; Gibbs \& Ng, 1977). We suggest that both of these conclusions are valid and not necessarily contradictory because working memory tested $6 \mathrm{hr}$ after training may reflect function of its longterm component. Furthermore, we assert that the Gibbs and $\mathrm{Ng}$ (1976) result can be construed as evidence for the hypothesis that protein synthesis is important for the long-term component of reference memory because the task they used can be considered a reference-memory-only task. When viewed in this way, the results reported here do not contradict those that suggest protein synthesis is important for formation of LTM but not STM. Instead, these results elaborate on that hypothesis.

Further research could be conducted to test the proposed interpretation of the data. 
A more stringent test of the separation of working and reference memory functions would be to adopt a procedure described by Olton, Becker, and Handelmann (1979). According to that procedure, only some of the arms are baited at the beginning of each trial, and the remaining arms are never baited throughout the experiment. The arms never baited test the function of reference memory, whereas performance on the baited arms reveals working memory function. On the basis of the results of this study, it would be expected that ANI would impair performance on baited (working memory) arms only if a sufficiently long delay were imposed between choices. In contrast, ANI should not affect performance on arms that were never baited.

Results from the three experiments reported here clearly demonstrate that ANI reduces choice accuracy by $20 \%-30 \%$. Although the impairment was statistically significant, it is interesting to note that performance was still well above the $40.2 \%$ correct expected by chance alone. Perhaps a higher dose of ANI would have resulted in a more complete amnesia. Results from ongoing experiments suggest this is not the case. Specifically, pretrial injections of ANI $(160 \mu \mathrm{g} / \mu \mathrm{l})$ result in a reduction in choice acccuracy after a 15 - or 240-min delay similar to that shown following injection of 80 $\mu \mathrm{g}$ of ANI. It is important, however, that $160 \mu \mathrm{g}$ of ANI did not affect retention after a 2 -min delay, a result indicating that the temporal gradient of development of amnesia is the same for the two doses.

Most of the research on the role of protein synthesis in memory formation has been carried out with tasks involving negative reinforcement. As a result, there has been some concern whether protein synthesis inhibitors can also block memory for positive reinforcement (Rosenzweig \& Bennett, 1984). The experiments described in the present article provide very clear evidence that protein synthesis inhibitors, such as ANI, can induce amnesia in an appetitive task. The impairment in choice accuracy cannot be attributed to aversive properties of the drug for two reasons. First, ANI-treated rats readily consumed food pellets encountered on the maze following a delay. Second, if ANI produced a conditioned place aversion to those arms entered before the delay (or to the food in those arms), performance after the delay should not have been impaired. Instead, rats given ANI should have preferred to enter only those arms not chosen before the delay. Because ANI-treated rats did not show such a preference but instead made errors by revisiting arms chosen before the delay, it is clear that the impairment in choice accuracy cannot be attributed to aversive properties of ANI.

The approach of conducting experiments to compare and contrast existing theories of dichotomized memory has been recently suggested by Olton (1983). The purpose for the comparison in his model was to determine similarities between human and animal work, both behaviorally and neurochemically, by contrasting working and reference memories with episodic and semantic memories and procedural and declarative memories (Cohen \& Squire, 1980). The purpose of the comparison proposed here is more specific: to compare existing hypotheses of memory formation processes in animals. Until we understand more clearly what and how animals learn, on both a cognitive and a neurochemical level, one should compare animal and human memories with caution. The hypothesis suggested here, although speculative, not only provides a working model by which to test the generalizability of previously proposed animal models but also allows more direct assessment of their functional significance.

\section{References}

Agranoff, B. W. (1967). Memory and protein synthesis. Scientific American, 216(6), 115-122.

Bennett, E. L., Rosenzweig, M. R., \& Flood, J. F. (1977). Protein synthesis and memory studies with anisomycin. In S. Roberts, A. Lajtha, \& W. Gispen (Eds.), Mechanisms, regulation and special function of protein synthesis in the brain (pp. 319-330). Amsterdam: Elsevier/North Holland Biomedical Press.

Bierley, R. A., Kesner, R. P., \& Novak, J. M. (1983). Episodic long-term memory in the rat: Effects of hippocampal stimulation. Behavioral Neuroscience, 97, 42-48.

Cohen, H. D., \& Barondes, S. H. (1968) Effect of acetoxycycloheximide on learning and memory of a light-dark discrimination. Nature, 218, 271-273.

Cohen, N. J., \& Squire, L. R. (1980). Preserved learn- 
ing and retention of pattern analyzing skill in amnesia: Dissociation of knowing how and knowing that. Science, 210, 207-209.

Daniels, D. (1972). Effects of acetoxycycloheximide on appetitive learning and memory. Quarterly Journal of Experimental Psychology, 24, 102-114.

Davis, H. P., Rosenzweig, M. R., Jones, O. J., \& Bennett, E. L. (1981). Inhibition of cerebral protein synthesis does not prolong short-term memory. Journal of Comparative and Physiological Psychology, 95, 556-564.

Gibbs, M. E., \& Ng, K. T. (1976). Memory formation: A new three-phase model. Neuroscience Letters, 2, 165-169.

Gibbs, M. E., \& Ng, K. T. (1977). Psychobiology of memory: Towards a model of memory formation. Biobehavioral Reviews, 1, 113-136.

Gibbs, M. E., \& Ng, K. T. (1978). Memory formation for an appetitive visual discrimination task in young chicks. Pharmacology Biochemistry and Behavior, 8, 271-276.

Gibbs, M. E., \& Ng. K. T. (1979). Behavioural stages in memory formation. Neuroscience Letters, 13,279 283.

Gibbs, M. E., \& Ng, K. T. (1984). Hormonal influences on the duration of short-term and intermediate stages of memory. Behavioural Brain Research, 11, 109-116.

Grant, D. S. (1982). Stimulus control of information processing in rat short-term memory. Journal of Experimental Psychology: Animal Behavior Processes, 8, 154-164.

Grecksch, G., \& Matthies, H. (1980). Two sensitive periods for the amnesic effect of anisomycin. Pharmacology Biochemistry and Behavior, 12, 663-665.

Grecksch, G., Ott, T., \& Matthies, H. (1980). The effect of intrahippocampally applied anisomycin on the retention of brightness discrimination in rats. Behavioral and Neural Biology, 29, 281-288.

Handelmann, G. E., \& Olton, D. S. (1981). Spatial memory following damage to hippocampal CA3 pyramidal cells with kainic acid: Impairment and recovery with preoperative training. Brain Research, $217,41-58$.

Honig, W. K. (1978). Studies of working memory in the pigeon. In S. H. Hulse, H. Fowler, \& W. K. Honig (Eds.), Cognitive processes in animal behavior (pp. 211-248). Hillsdale, NJ: Erlbaum.

Keppel, G. (1982). Design and analysis: A researcher's handbook. Englewood Cliffs, NJ: Prentice-Hall.

Kesner, R. P., Partlow, L. M., Bush, L. G., \& Berman, R. F. (1981). A quantitative regional analysis of protein synthesis inhibition in the rat brain follow- ing localized injection of cycloheximide. Brain Research, 209, 159-176.

Klapp, S. T., Marshburn, E. A., \& Lester, P. T. (1983). Short-term memory does not involve the "working memory" of information processing: The demise of a common assumption. Journal of Experimental Psychology: General, 112, 240-264.

Maki, W. S., Brokofsky, S., \& Berg, B. (1979). Spatial memory in rats: Resistance to retroactive interference. Animal Learning \& Behavior, 7, 25-30.

Olton, D. S. (1978). Characteristics of spatial memory. In S. H. Hulse, H. Fowler, \& W. K. Honig (Eds.), Cognitive processes in animal behavior (pp. 341373). Hillsdale, NJ: Erlbaum.

Olton, D. S. (1983). Memory functions and the hippocampus. In W. Seifert (Ed.), Neurobiology of the hippocampus (pp. 335-373). New York: Academic Press.

Olton, D. S., Becker, J. T., \& Handelmann, G. E. (1979). Hippocampus, space, and memory. Behavioral and Brain Sciences, 2, 313-365. (Includes commentary)

Olton, D. S., Handelmann, G. E., \& Walker, J. A. (1981) Spatial memory and food searching strategies. In A. C. Kamil \& T. D. Sargent (Eds.), Foraging behavior: Ecological, ethological and psychological approaches (pp. 333-354). New York: STPM Press.

Olton, D. S., \& Samuelson, R. J. (1976) Remembrance of places passed: Spatial memory in rats. Journal of Experimental Psychology: Animal Behavior Processes, 2, 97-116.

Rawlins, J. N. P., \& Tsaltas, E. (1983). The hippocampus, time and working memory. Behavioral Brain Research, 10, 233-262.

Rosenzweig, M. R., \& Bennett, E. L. (1984). Basic processes and modulatory influences in the stages of memory formation. In G. Lynch, J. L. McGaugh, \& N. Weinberger (Eds.), Neurobiology of learning and memory (pp. 263-288). New York: Guilford Press.

Shavalia, D. A., Dodge, A. M., \& Beatty, W. W. (1981). Time-dependent effects of ECS on spatial memory in rats. Behavioral and Neural Biology, 31, 261-273.

Tulving, E. (1972). Episodic and semantic memory. In E. Tulving \& W. Donaldson (Eds.), Organization of memory (pp. 381-403). New York: Academic Press.

Wagner, A. (1978). Expectancies and the priming of STM. In S. H. Hulse, H. Fowler, \& W. K. Honig (Eds.), Cognitive processes in animal behavior (pp. 177-209). Hillsdale, NJ: Erlbaum.

Received February 17, 1984 Revision received June 25, 1984 